\title{
Review urges US physicists to think globally
}

\section{Irwin Goodwin, Washington}

In a major review of the entire field of physics, a panel of the US National Academy of Sciences has called on physicists to promote international collaboration, and to make more of the importance of applications of their work to the life sciences.

The panel's report, Physics in a New Era, urges Congress and federal agencies to develop new protocols for international cooperation. "A key issue is the importance of long-term, stable funding for large-scale international projects," it says.

The issue is critical to US physicists, following the collapse of the Superconducting Super Collider project in Waxahachie, Texas, in 1993, and the 1999 abandonment of US involvement in the International Thermonuclear Energy Reactor, a global collaboration in fusion research.

The call for collaboration is one of nine main recommendations in the report, to be released later this month. The panel also recommends that funding for basic physics be restored, as a share of the economy, to the level it enjoyed 20 years ago, requiring current spending to rise by about one-third.

It argues that "along with the high-tech economy, the biological and medical sciences have benefited enormously from this research. Such physics-invented technologies as X-ray crystallography, magnetic resonance, fibre optics, electron microscopy, mass spectroscopy and radioactive tracers are at the heart of the rapid pace of discovery in almost every biomedical laboratory."

The panel is mildly critical of university physics departments, urging them to review and revise their curricula, "and to reverse... the long-term decline in the numbers of undergraduate and graduate students studying physics".

It calls on science agencies that fund physics - the Department of Energy, the National Science Foundation, NASA and the Department of Defense - to support the work of more individual investigators. It says that funding for small groups and single investigators is "dangerously inadequate".

It also warns that security problems at the Department of Energy's nuclear-weapons laboratories have produced an adversarial climate between scientists and managers. "The morale of scientists is low, and recruitment and retention difficulties threaten the viability of the laboratories," the panel says.

The report is an overview of a ten-year study by the academy that has spawned six smaller reports on subdisciplines of physics. Unlike its influential decadal reviews of astronomy, for example, the academy's physics review does not set priorities for funding of specific facilities or projects.

"Ours is an order of magnitude different

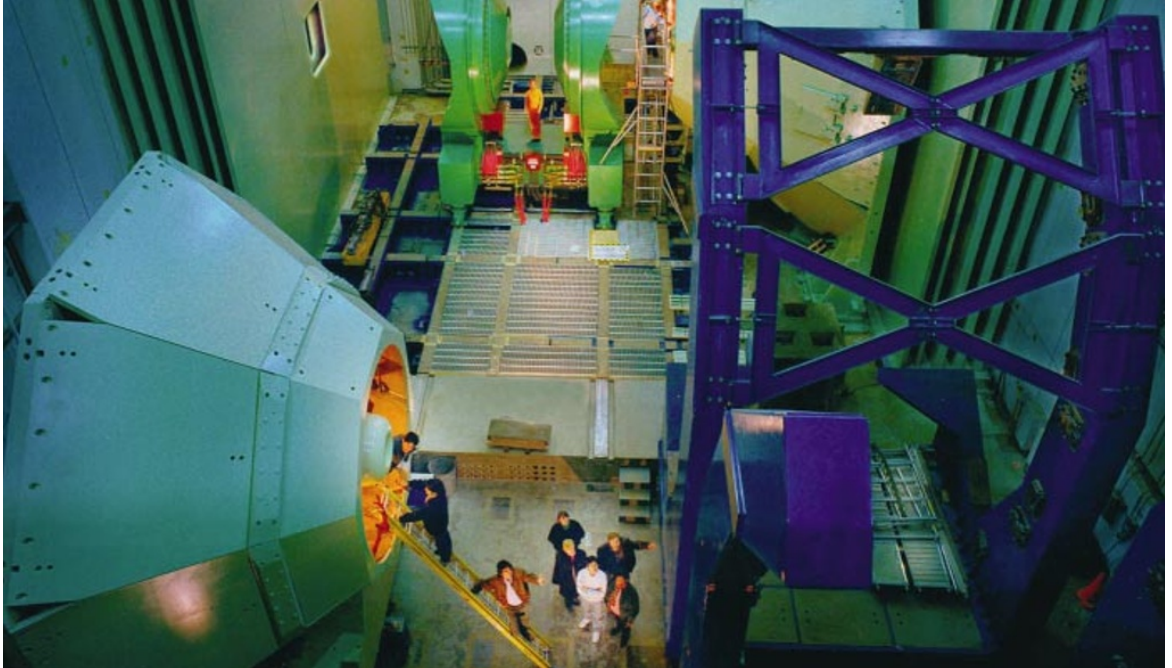

Local enterprise: new mechanisms are needed to enable international physics projects to match the success of US-only ones, such as Brookhaven's Relativistic Heavy Ion Collider (above).

from the astronomy reports," explains Yale University's Tom Appelquist, chairman of the 14-member panel. "We speak about the opportunities for discovery that cut across the expanding diversity of physics. Our priorities are not a wish list."

"It's devilishly difficult and divisive to set priorities in a report that encompasses all of physics," says James Langer of the University of California, Santa Barbara, last year's president of the American Physical Society.

The earlier subpanel reports did endorse particular projects: particle physicists, for example, recommended capitalizing on the potential of Fermilab's main injector. Nuclear physicists supported Brookhaven's Relativistic Heavy Ion Collider and promoted an as-yet-unbuilt rare-isotope accelerator, and condensed-matter physicists championed the Spallation Neutron Source in Oak Ridge, Tennessee.

The overall report calls on US physicists to "develop a broadly shared vision" of experimental research facilities and to "communicate this vision clearly and persuasively" to politicians and the public.

\section{MIT to set up Media Lab in India}

\section{K. S. Jayaraman, New Delhi}

The Indian government has approved a ten-year, \$1.1-billion joint project with the Massachusetts Institute of Technology (MIT) to set up Media Lab Asia, modelled on the highly successful Media Lab in Cambridge, Massachusetts.

The Indian facility will be MIT's second Media Lab outside the United States, following the establishment last year of Media Lab Europe in Dublin, Ireland.

Announcing the agreement on 31 May, Indian information-technology minister Pramod Mahajan said that MIT had chosen India after evaluating offers from several other Asian countries, including China and Singapore. A formal agreement between his ministry and MIT is expected to be signed this month.

Mahajan said the project would initially have a one-year "exploratory phase", for which the Indian government has committed \$14 million. Whether it continues for the next nine years will then depend on an end-of-year review.

If it continues, the government will provide $20 \%$ of the funding, with the rest expected to come from industry. Mahajan said the goal is to make the new laboratory self-sustainable, with income from the commercialization of its research findings.

Although conceptually similar to its cousins in the United States and Europe, Media Lab Asia will have a more decentralized structure, with offices in Mumbai, New Delhi and Bangalore. Mahajan says it will focus on technologies and products that are of specific value to Asia.

In particular, it will develop ideas for future global and domestic markets, and seek affordable solutions to problems of health education and rural development. One of its first tasks will be to identify academic partners to work with entrepreneurs on projects to benefit rural areas. 